\title{
Stimuli-Responsive Biomimetic Metallic Luster Film Using Dye Absorption and Specular Reflection from Layered Microcrystals
}

Yuki Kojima ${ }^{\dagger}$, Keiki Kishikawa ${ }^{\dagger}$, Shuji Ichikawa ${ }^{\ddagger}$, Jun Matsui ${ }^{\S}$, Keita Hirai ${ }^{\perp}$, Yukishige Kondo", and Michinari Kohri*†

$\dagger$ Department of Applied Chemistry and Biotechnology, Graduate School of Engineering, Chiba University, 1-33 Yayoi-cho, Inage-ku, Chiba 263-8522, Japan

$\$$ Yokohama Research \& Development Center, Mitsubishi Pencil Co., Ltd., 2-5-12 Irie, Kanagawa-ku, Yokohama 221-8550, Japan

$\S$ Faculty of Science, Yamagata University, Kojirakawa-cho, Yamagata, 990-8560, Japan

${ }^{\perp}$ Department of Imaging Sciences, Graduate School of Engineering, Chiba University, 1-33 Yayoi-cho, Chiba, 263-8522, Japan

" Department of Industrial Chemistry, Faculty of Engineering, Tokyo University of Science, 13 Kagurazaka, Shinjuku, Tokyo, 162-8601, Japan

Corresponding author

*E-mail: kohri@faculty.chiba-u.jp (M. K.) 
(a)
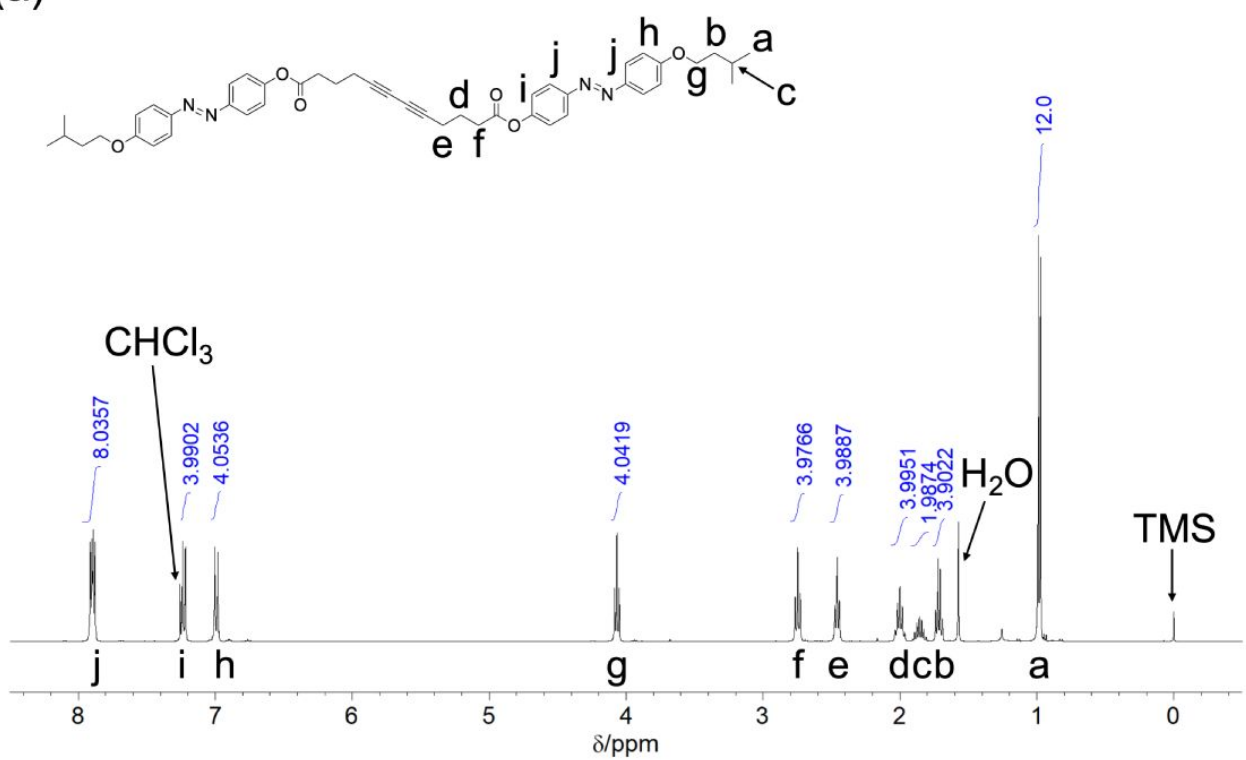

(b)

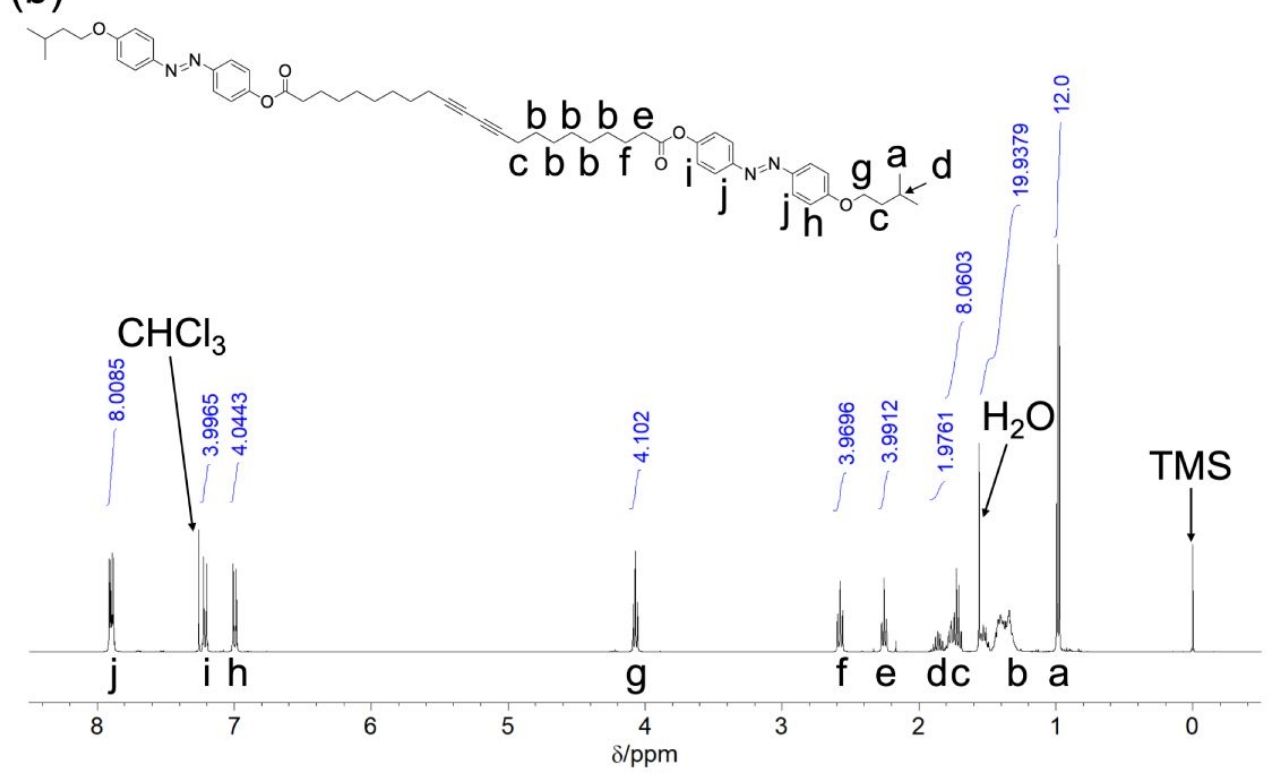

Figure S1. ${ }^{1} \mathrm{H}$ NMR spectra of (a) DAzo-DA1 and (b) DAzo-DA2 in $\mathrm{CDCl}_{3}$. 
(a)
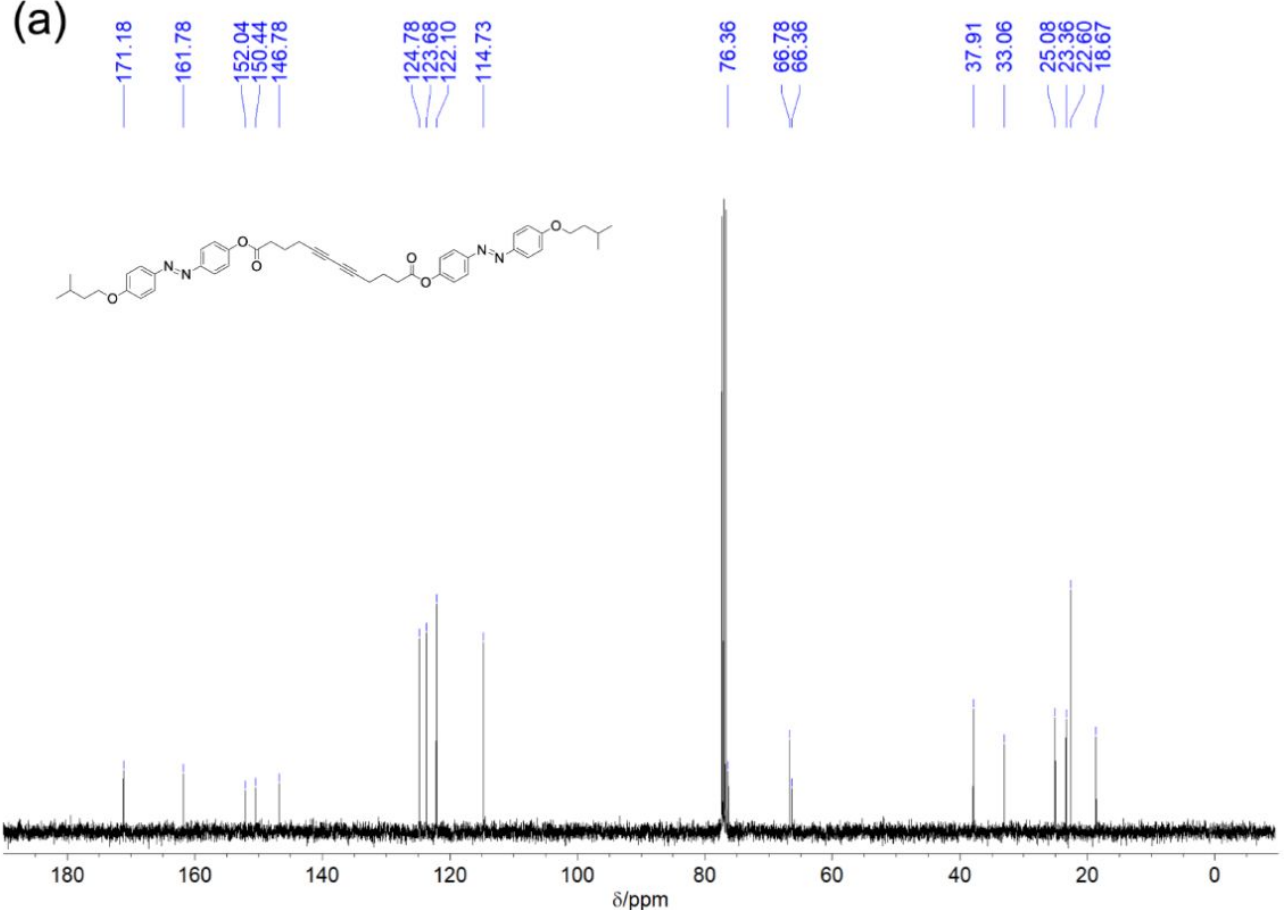

(b)

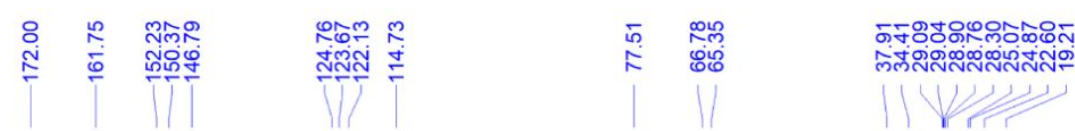

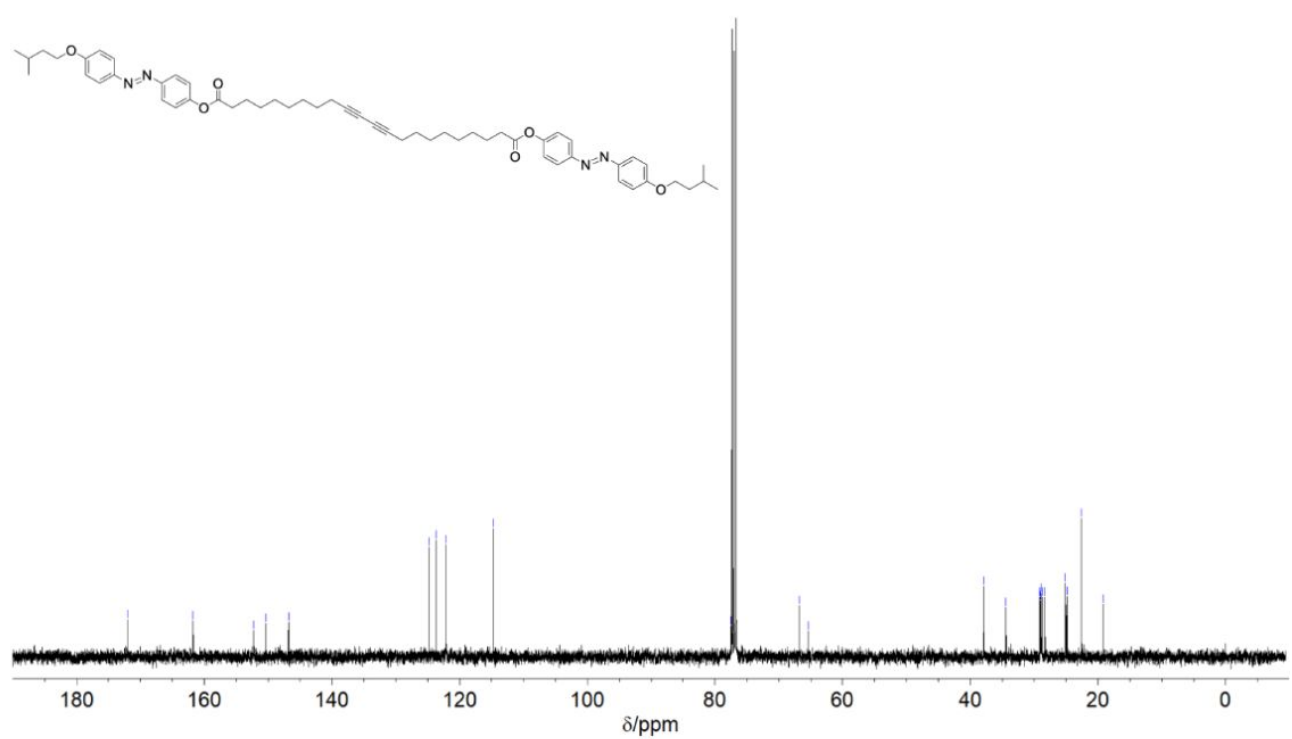

Figure S2. ${ }^{13} \mathrm{C}$ NMR spectra of (a) DAzo-DA1 and (b) DAzo-DA2 in $\mathrm{CDCl}_{3}$. 
(a)

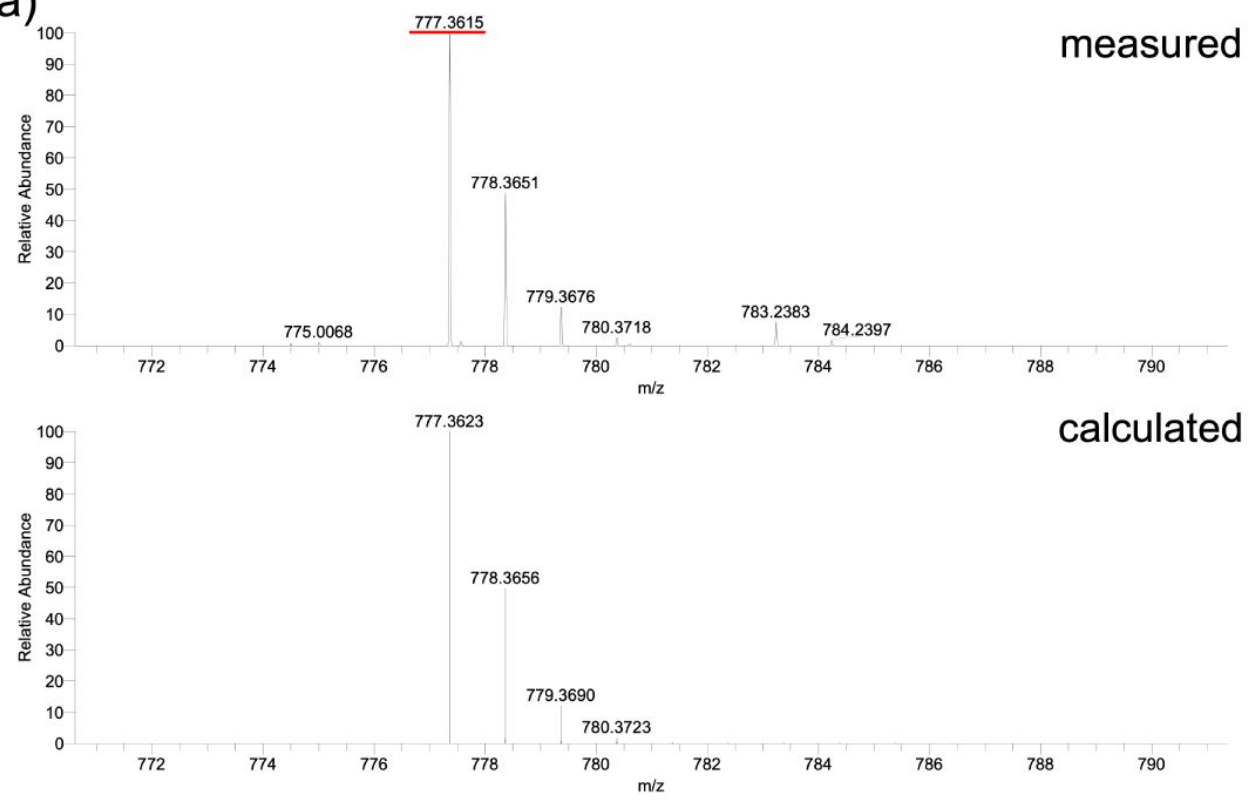

(b)
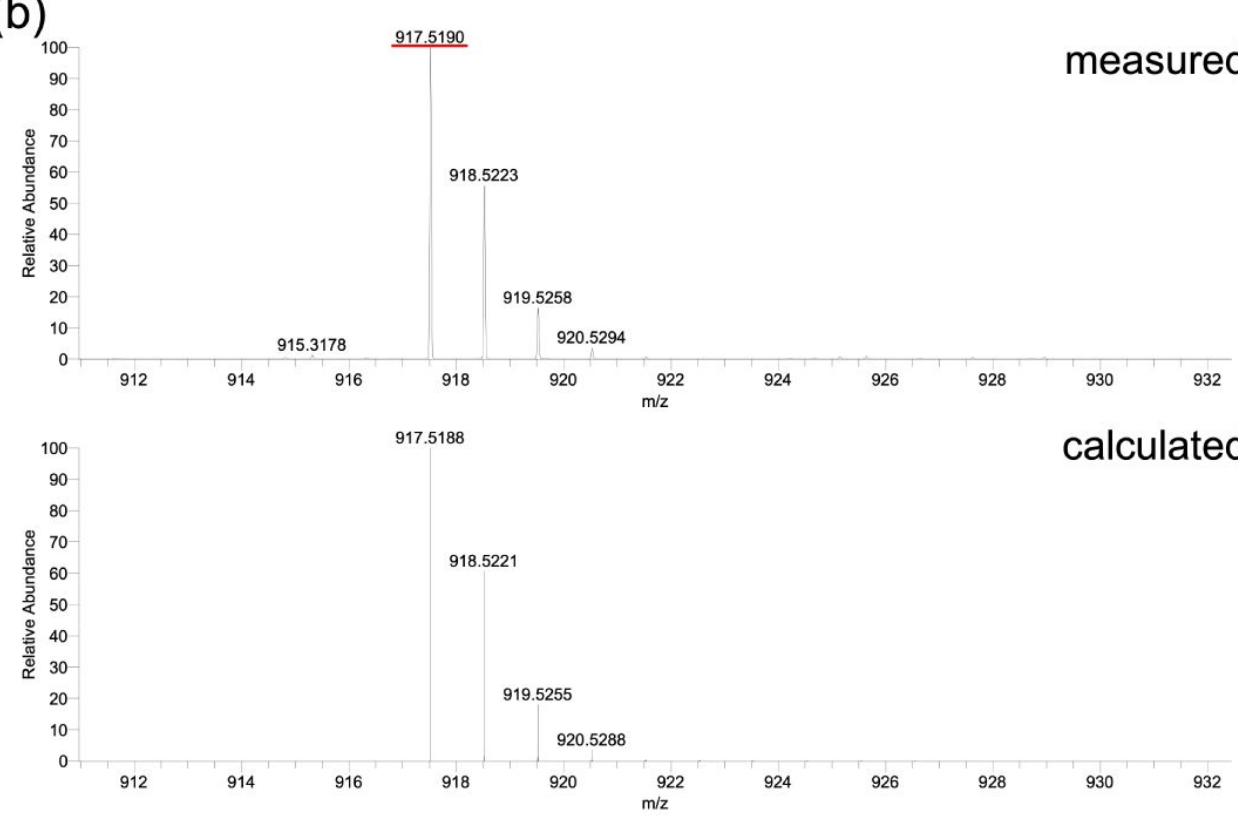

Figure S3. MS spectra of (a) DAzo-DA1 and (b) DAzo-DA2. 
(a)

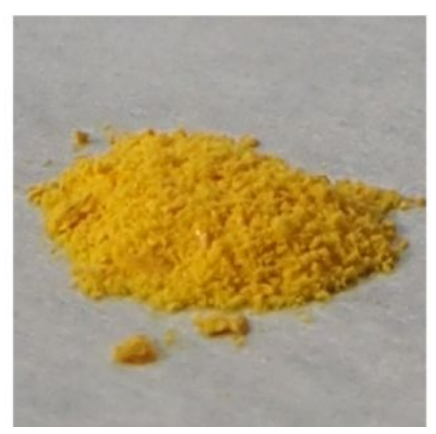

(b)

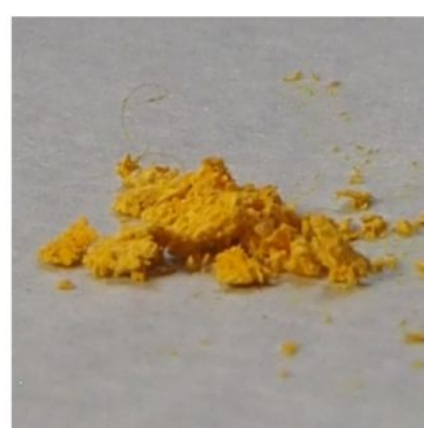

Figure S4. Photographs of powder samples of (a) DAzo-DA1 and (b) DAzo-DA2.
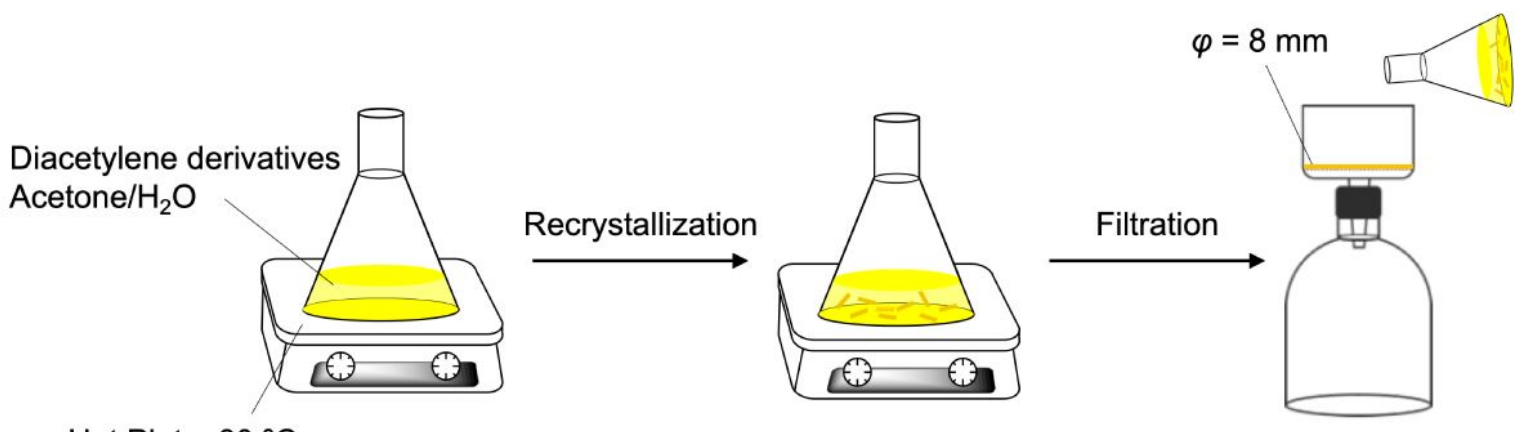

Hot Plate: $80^{\circ} \mathrm{C}$

Figure S5. Schematic illustration of the preparation of diacetylene derivative crystal films through recrystallization and filtration.

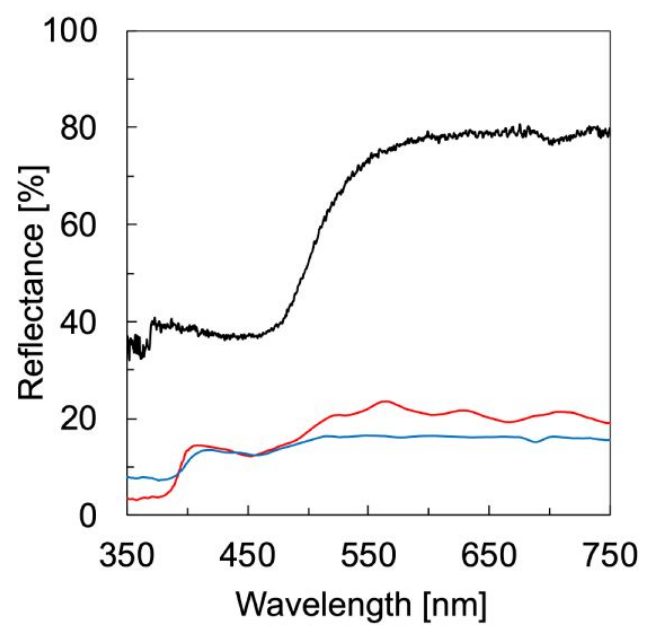

Figure S6. Reflection spectra of DAzo-DA1-film (red line), DAzo-DA2-film (blue line), and gold leaf (black line). 
(a) thickness

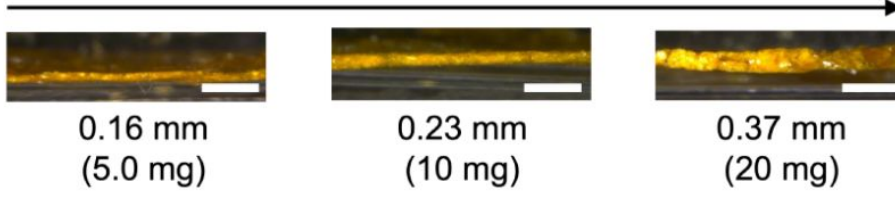

(b)
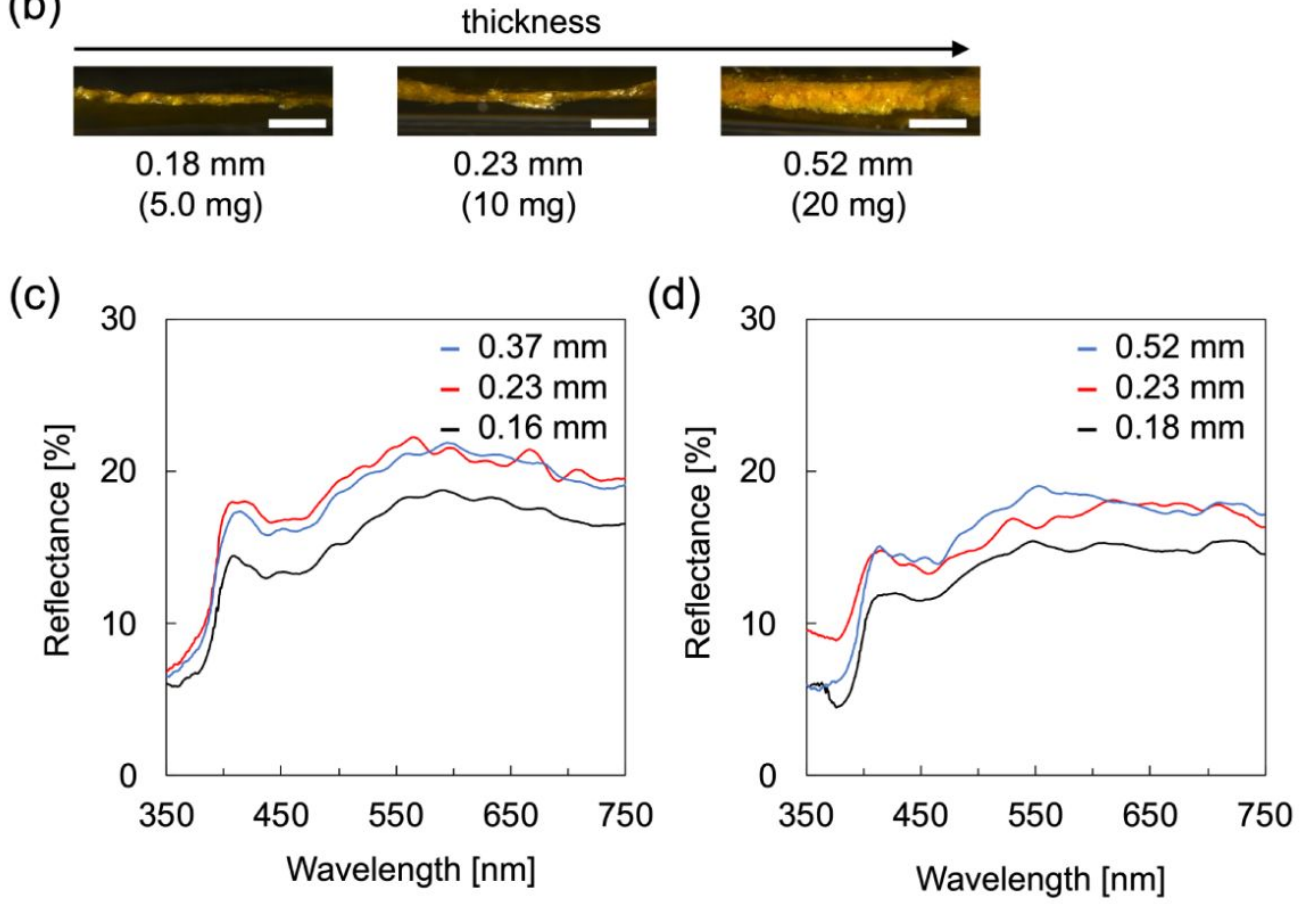

Figure S7. Photographs of the side view of (a) DAzo-DA1-film and (b) DAzo-DA2-film with different thicknesses. Scale bars are $0.5 \mathrm{~mm}$. The numbers indicate the average film thickness and the numbers in parentheses indicate the amount of sample used for recrystallization. Reflection spectra of (c) DAzo-DA1-film and (d) DAzo-DA2-film with different thicknesses.
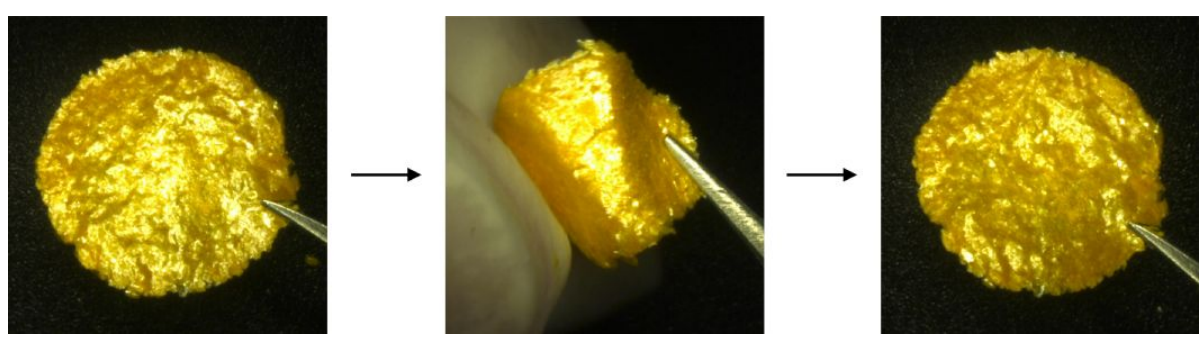

Figure S8. Demonstration of bending test for the free-standing DAzo-DA1-film (film thickness: $0.23 \mathrm{~mm}$ ) picked with tweezers. 

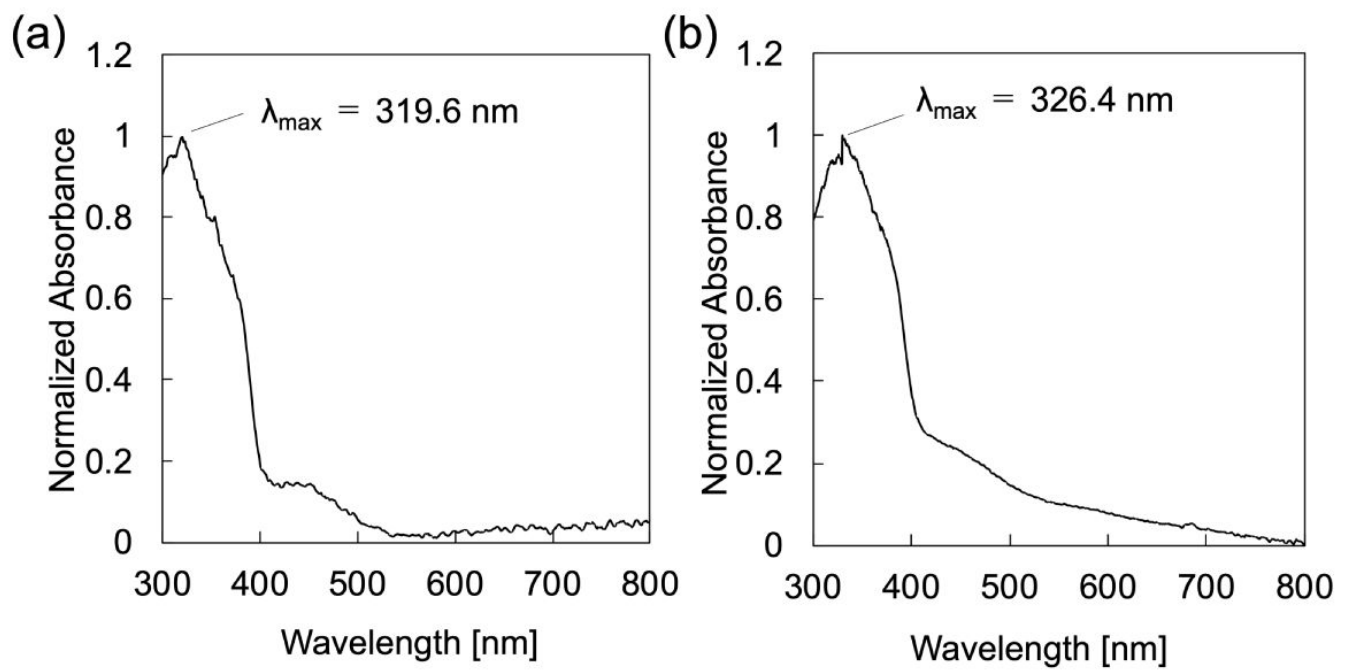

Figure S9. UV-vis absorption spectra of (a) DAzo-DA1-film and (b) DAzo-DA2-film.
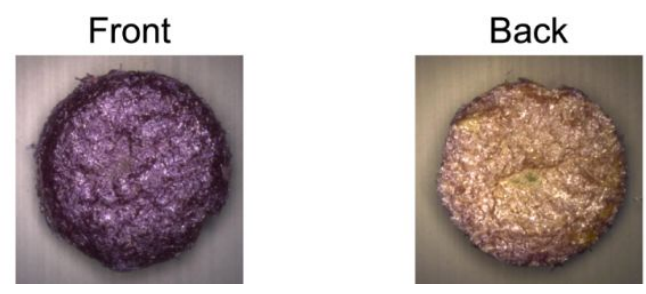

Figure S10. Photographs of the front and back surfaces of DAzo-DA2(UV)-film (film thickness: $0.23 \mathrm{~mm}$ ) after UV irradiation $(254 \mathrm{~nm})$.

(a)

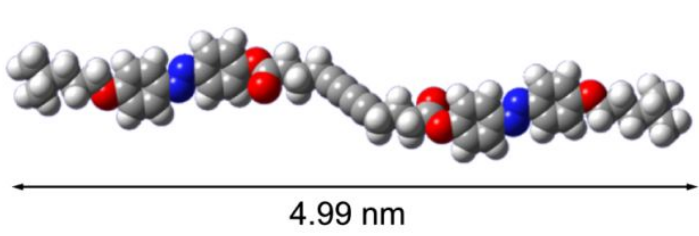

(b)

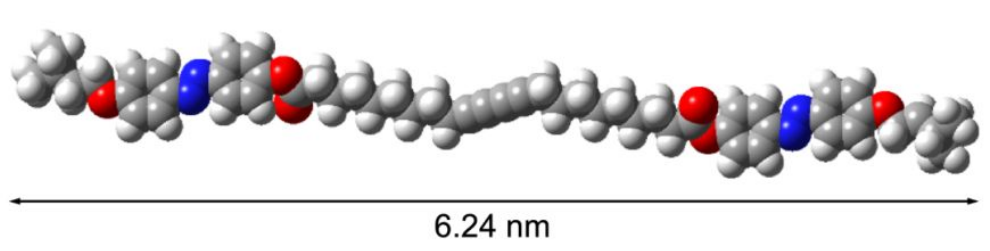

Figure S11. Molecular models of (a) DAzo-DA1 and (b) DAzo-DA2. Molecular length was determined from the end-to-end distance of the optimized molecular models calculated from DFT calculation (B3LYP/6-31G(d)). 
(a)

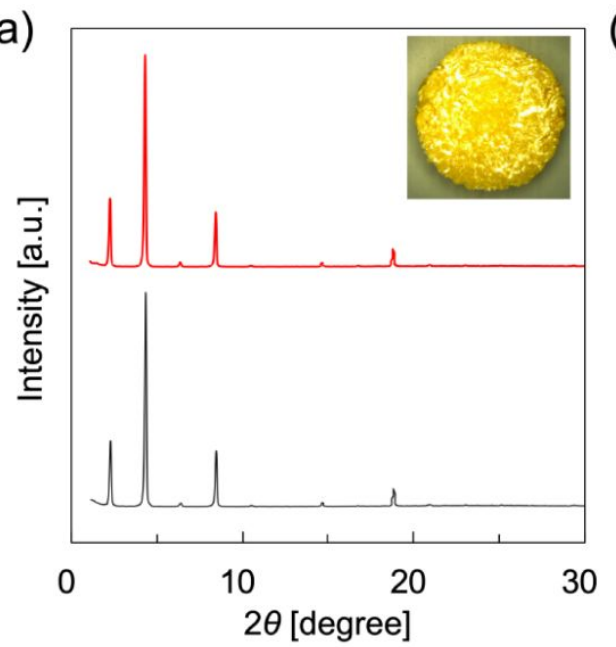

(b)

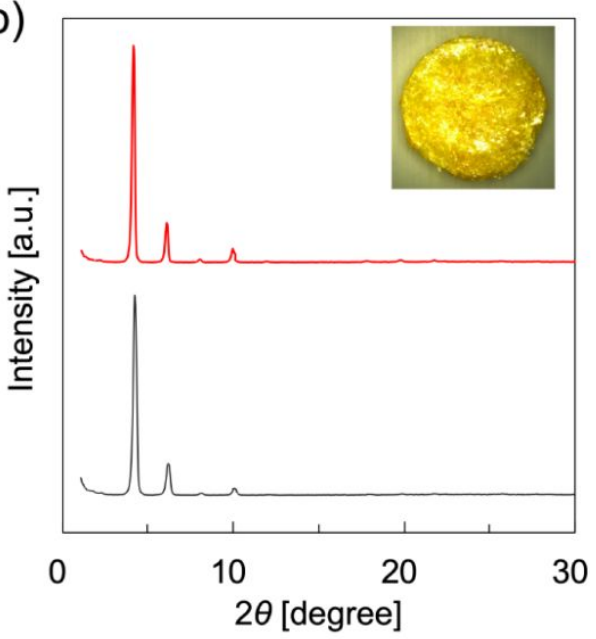

Figure S12. XRD patterns of (a) DAzo-DA1-film and (b) DAzo-DA2-film before (black lines) and after (red lines) UV irradiation $(365 \mathrm{~nm})$. The insets show the photographs of DAzo-DA1film and DAzo-DA2-film after UV irradiation (365 nm).

(a)

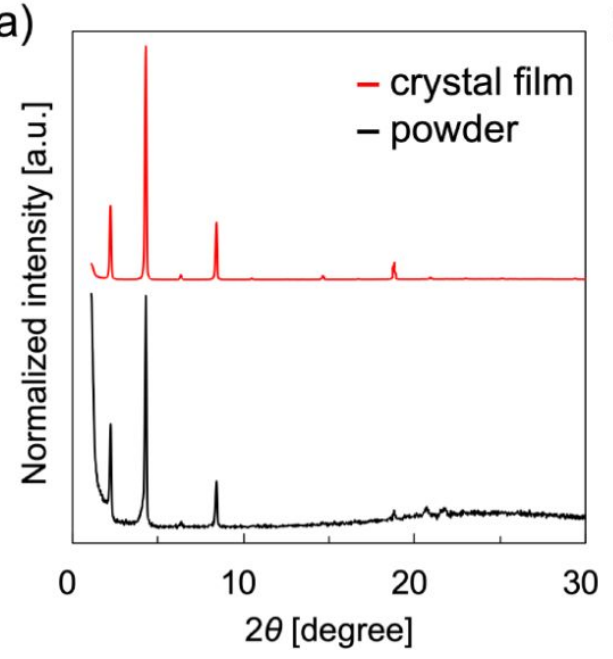

(b)

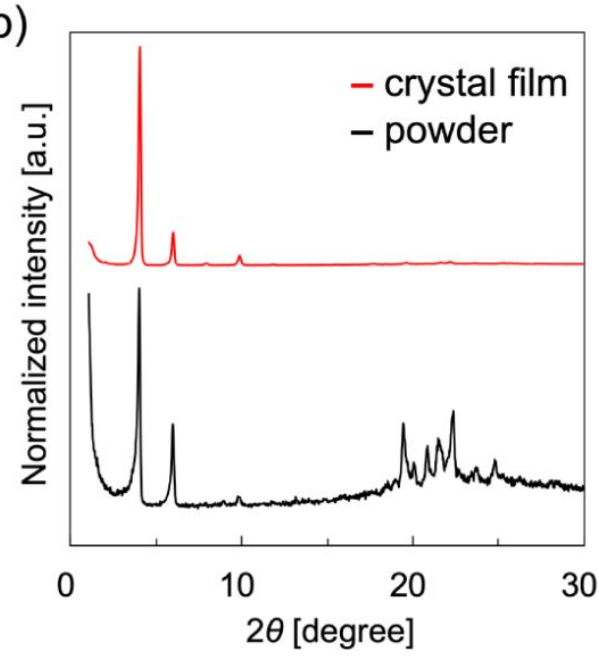

Figure S13. XRD patterns of crystal film (red lines) and powder (black lines) of (a) DAzoDA1 and (b) DAzo-DA2. 